\title{
Accuracy of Bony Resection under Computer Assisted Navigation Compared with Free-hand Cutting for Bone Tumors around the Knee
}

\author{
Zhiping Deng, Bin Li, Tao Jin, Qing Zhang, Lin Hao, Xiaohui Niu* \\ Department of Orthopaedic Oncology Surgery, Beijing Jishuitan Hospital, Peking University, \\ Beijing, 100035, China \\ *Corresponding author: Prof Xiaohui Niu, niuxiaohui@,263.net
}

\section{Introduction}

Bone tumor surgery can be more accurate under the computer assisted navigation. The researches have shown the benefit of margin control in pelvic, joint sparing surgery [1-3]. The traditional method for bone cutting in limb salvage was by free hand. There was no literature focused on the comparison of cutting accuracy in bone tumors around the knee joint. The aim of this study was to compare the accuracy for bony resection under navigation and by free hand in limb salvage surgery around the knee.

\section{Materials and Methods}

Thirty-nine cases of bone tumors around the knee joint were resected under navigation (Stryker System) in our department from 2008 Sep to 2017 Nov. All the cases were performed intercalary resection in femur or tibia. The initial aim to use navigation was to make the resection more close to the pre-operative planning. The pre-operative planning was performed with the software OrthoMap at the working station (Stryker Company). The CT and MRI imaging were input to the system. We used intraoperative navigation (Iso-C based) to find the cutting plane and use the jig saw to cut the bone. 
The post-operative specimen was used for verification and compared with the pre-operative plan. The length difference was defined as specimen length minus planning resection length. The control group included 117 cases of tumors around the knee performed limb salvage surgery when bony cutting was achieved by free hand. This was a non-randomized control study. The method to find the cutting plane was by measuring the length from the joint line to the planned plane by ruler. The length of post-operative specimen by free hand was compared with the surgical plan. The length difference was detected in this control group. Then the differences in two groups were compared and analyzed.

\section{Results}

The resection lengths in navigation group ranged from $85-282 \mathrm{~mm}$ and in the free hand group the length ranged from $90-330 \mathrm{~mm}$. The length difference between post-operative verification and pre-operative plan was detected. In the navigation group, the length difference was $0.5 \pm 2.5 \mathrm{~mm}$ (range , $-5 \sim 5 \mathrm{~mm}$ ), while in the free hand group the length difference was $3.4 \pm 9.6 \mathrm{~mm}$ (range ,-20 $29 \mathrm{~mm}), \mathrm{P}<0.01$. For the absolute value differences analysis, the difference was $2.0 \pm 1.6 \mathrm{~mm}$ and $8.3 \pm 6.0 \mathrm{~mm}$ for navigation and free hand group respectively, $\mathrm{P}<0.01$.

\section{Discussion}

Our study shows that bone cutting with navigation can be more accurate than freehand cutting. The average length difference was $2.0 \mathrm{~mm}$ ( $95 \% \mathrm{CI}, 0.4$ to $3.6 \mathrm{~mm}$ ) when compared to average $8.3 \mathrm{~mm}$ (95\% CI, 2.3 to $14.3 \mathrm{~mm})$. The accuracy with navigation is similar to the previous researches [4-5]. Our comparison with free hand group gives the data how accurate the navigation can help surgeon to achieve. The result indicates that computer assisted navigation can make a role in limb salvage surgery if the precise resection is required.

\section{References}

1.Jeys L, Matharu GS, Nandra RS, et al.Can computer navigation-assisted surgery reduce the risk of an intralesional margin and reduce the rate of local recurrence in patients with a tumour of the pelvis or sacrum? Bone Joint J. 2013 Oct;95-B(10):1417-24.

2.Cartiaux O, Banse X, Paul L, et al.Computer-assisted planning and navigation improves cutting accuracy during simulated bone tumor surgery of the pelvis.Comput Aided Surg. 2013;18(1-2):19-26

3.Wong KC, Kumta SM.Joint-preserving tumor resection and reconstruction using image-guided computer navigation.Clin Orthop Relat Res. 2013 Mar;471(3):762-73.

4.Ritacco LE, Milano FE, Farfalli GL,et al.Accuracy of 3-D planning and navigation in bone tumor 
resection.Orthopedics. 2013 Jul;36(7):e942-50

5.So TY, Lam YL, Mak KL.Computer-assisted navigation in bone tumor surgery: seamless workflow model and evolution of technique.Clin Orthop Relat Res. 2010 Nov;468(11):2985-91

\section{Disclosures}

None 